\title{
Investigation of parameters influencing erosive wear using DEM
}

\author{
Akbar JAFARI ${ }^{*}$, Reza ABBASI HATTANI \\ Department of Mechanical Engineering, Sirjan University of Technology, Sirjan 7813733385, Iran \\ Received: 15 September 2017 / Revised: 08 May 2018 / Accepted: 28 October 2018 \\ (C) The author(s) 2018. This article is published with open access at Springerlink.com
}

\begin{abstract}
The effects of several parameters on the erosive wear were studied using the discrete element method (DEM). The Finnie model was implemented using an open-source code. Regarding the time integration, the Gear algorithm was used, and to ensure the accuracy of the DEM results, a time-step sensitivity analysis was performed. The problem was modeled in two parts: first, the impact of a single particle on a surface was modeled, and then a more general model was prepared to examine the wear of surfaces under the flow of particles. The effects of the surface area, impact angle, speed, particle size, particle density, Young's modulus, Poisson's ratio, and restitution coefficient on the wear were studied numerically, and the results are discussed herein.
\end{abstract}

Keywords: wear; erosion; Finnie; discrete element

\section{Introduction}

Wear is among the important issues from the theoretical, practical, economic, and environmental viewpoints. For instance, approximately $3 \%$ of the world's total energy is used to remanufacture parts that fail owing to wear [1]. Therefore, there are sufficient motivations for scientific and research investment in this field. Because of the complexity of the issue, it is difficult to be modeled theoretically and predicted practically. No consensus exists in the literature regarding the classification of wear. For instance, in Ref. [2], the wear is categorized into four main types-abrasive, adhesive, corrosive, and surface fatigue-and the others, such as erosion, are classified as minor types. As another tactic in Ref. [3], the surface state, interaction mechanism, and relative motion were considered as three main indices for categorizing wear, and a unified wear classification was proposed [3]. A literature review indicates that several studies have been conducted in this field, especially using experimental methods. The experimental study performed by Archard [4] is fundamental research that serves as a cornerstone to wear analysis. Lynn et al. [5] employed an experimental approach for understanding the effects of the particle size inside a slurry on the flow erosive wear. They concluded that the wear rate is related to the kinetic energy of the particles. They proved that if the kinetic energy exceeds a threshold, it causes surface failure and wear [5]. Chacon-Nava et al. [6] used a rubber wheel tester to study the effect of the surface hardness and particle size on the abrasive wear. In the particle-size range that they tested, smaller particles led to larger wear. Such behavior is linked to the cutting effect of small particles, while large particles cause plastic deformation [6]. According to the results of Ojala et al. [7], the particle size affects the stress level, but no absolute conclusion can be drawn regarding the effects of the particle size. In this regard, they found that when the particle size was less than $1 \mathrm{~mm}$, a low-stress condition occurred, and the tested elastomers showed better wear resistance than the tested steels [7]. According to their results, the dependency of the wear on the particle size can exhibit both ascending and descending trends [7]. Woldman et al. [8] studied the effects of particle properties on the wear of parts that

* Corresponding author: Akbar JAFARI, E-mail: jafari@sirjantech.ac.ir 


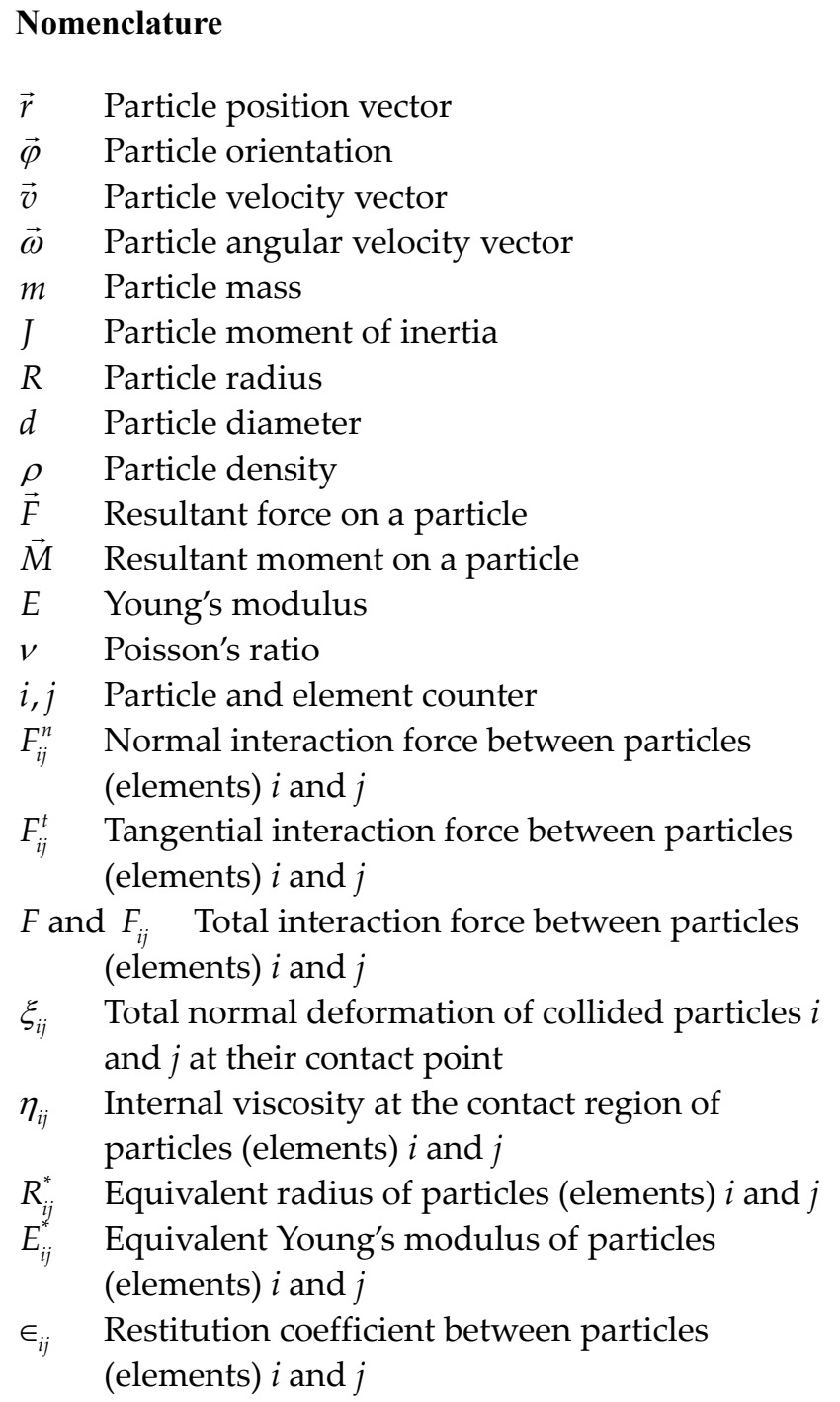

$K_{i j}^{n} \quad$ Normal equivalent spring between particles (elements) $i$ and $j$

$v_{i j}^{t} \quad$ Relative tangential velocity at the contact point of particles (elements) $i$ and $j$

$\gamma_{i j}^{t} \quad$ Viscous friction coefficient between the interacting particles (elements) $i$ and $j$

$\mu_{i j} \quad$ Dry friction coefficient between the interacting particles (elements) $i$ and $j$

$W \quad$ Removed volume from a point (wear)

$w$ Rate of the wear

$K_{\mathrm{F}} \quad$ Finnie wear constant

$\alpha \quad$ Impact angle

$Z \quad$ Function for the dependency of the erosion on the impact angle

$k \quad$ Conditional constant in the Finnie wear model

$t \quad$ Time

$t_{\mathrm{p}} \quad$ Time of penetration during a collision

$\vec{q} \quad$ Unit vector pointing from the particle center to the contact point

$H$ Heaviside function

A Surface area of the single-particle model

$\alpha_{c} \quad$ Impact angle at which maximum wear occurs

$\beta \quad$ Angle of the sample inside the particle flow

$C$ Coefficient for the dependence of the wear on the speed

$n \quad$ Power constant for the dependence of the wear on the speed operate in sandy environments. Although they showed the importance of the particle size and shape, no implicit relation was observed [8]. Many other studies have employed experimental methods; however, theoretical approaches have also been developed to study wear.

Finnie proposed a mathematical model to predict the wear under the impact of particles [9]. The mass, speed, and impact angle of the particles play roles in this model. Other analytical models, such as those of Archard [10], Wellinger et al. [11], Hutchings [12], and Rabinowicz [13], have also been proposed. The finite-element method has been employed as a conventional numerical method to model wear; however, this method has generally been applied to study the abrasive and adhesive wear mechanisms [14-17]. On the other hand, the discrete element method (DEM) is becoming favorable in this field, as it is able to apply the introduced mathematical models under complex conditions. The study of Cleary [18] is among the first published reference in the field of using DEM for wear analysis and focused on mill liners. Kalala et al. $[19,20]$ studied the wear of mill liners using the DEM. They analyzed the effects of the liner profile on the wear regime. Ashrafizadeh et al. [21] used the DEM to study the effects of the impact angle and particle energy on the wear of a surface. They showed that the maximum shear impact energy occurs at an impact angle of approximately $30^{\circ}$ and concluded that this angle causes the highest wear. However, we believe 
that this conclusion is limited to materials with a low shear strength. Zhang et al. [22] and Tan et al. [23] used the DEM to study the erosive wear in pipes carrying a slurry. They showed that the effect of the speed on the wear rate in the elbow region was larger than that in the straight parts and determined the critical region of the elbow. On the same topic, Uzi et al. [24] explored variations of the wear in different regions of the elbow in conveying pipelines. Powell et al. [25] used the DEM to determine the impact energy of particles in a mill. They estimated the profile of the liners undergoing wear gradually. Varga et al. [26] employed both an experimental approach and the DEM to evaluate the wear in pipes carrying a slurry. As another case study, Jafari et al. employed the DEM to examine the effects of the vibration screen characteristics on its mesh wear [27]. Chu et al. [28] used the DEM to predict the wear in dense medium cyclones (DMCs) and proposed this approach for studying effects of the wear on the DMC performance. Forsström et al. [29] employed the DEM with Archard's wear model, finding that the critical region of tipper bodies ruptured owing to abrasive wear. They concluded that such numerical simulations are useful for optimizing the tipper body geometry in order to avoid local failure and improve its service life.

According to the foregoing literature review, the DEM has the potential to model wear; nonetheless, this field is not fully developed, and further investigations can be valuable for enhancing the modeling of the wear under realistic conditions. Moreover, this can assist researchers to reduce the requirements of experimental setups, which are generally expensive and take considerable time. In this regard, the present study employs the DEM to investigate the parameters that affect the wear following two procedures. In the first part, a single-particle model is used to observe the basics of the wear due to the impact of a particle, and in the second part, simulations inspired by a mixture-type experimental setup are performed.

\section{DEM}

According to the DEM, motion equations are solved to determine the position, velocity, and acceleration of all the particles in the system. For a system of $N$ particles, the equilibrium equations of the force and moment for the $i^{\text {th }}$ particle are as follows:

$$
\begin{aligned}
& \frac{\partial^{2} \vec{r}_{i}}{\partial t^{2}}=\frac{1}{m_{i}} \vec{F}_{i}\left(\vec{r}_{i}, \vec{v}_{i}, \vec{\varphi}_{i}, \vec{\omega}_{i}\right), \quad(i=1, \ldots, N) \\
& \frac{\partial^{2} \vec{\varphi}_{i}}{\partial t^{2}}=\frac{1}{J_{i}} \vec{M}_{i}\left(\vec{r}_{i}, \vec{v}_{i}, \vec{\varphi}_{i}, \vec{\omega}_{i}\right), \quad(i=1, \ldots, N)
\end{aligned}
$$

where $\vec{r}_{i}$ is the position vector, $\vec{\varphi}_{i}$ is the orientation, $\vec{v}_{i}$ is the velocity, $\vec{\omega}_{i}$ is the angular velocity, $m_{i}$ is the mass, and $J_{i}$ is the moment of inertia of the $i^{\text {th }}$ particle. $\vec{F}_{i}$ and $\vec{M}_{i}$ are the resultant force and moment on the $i^{\text {th }}$ particle, respectively, and are determined as follows,

$$
\begin{aligned}
& \vec{F}_{i}=\sum_{j=1, j \neq i}^{N} \vec{F}_{i j} \\
& \vec{M}_{i}=\sum_{j=1, j \neq i}^{N} \vec{M}_{i j}
\end{aligned}
$$

Here, $\vec{F}_{i j}$ and $\vec{M}_{i j}$ are the interacting force and moment, respectively, acting from particle or element $j$ on particle $i$. The interaction force arises from direct contact between particles and contact between particles and surface elements. On the other hand, the moment is due to the action of the tangential force on the particle. The equation (s) for the estimation of the tangential force will be provided subsequently. Several models have been proposed for estimating the interaction forces between contacting particles [27]. The viscoelastic Kelvin-Hertz model employed here relates the normal interaction force to the normal deformation and deformation rate, as follows [30]. According to this model, the interaction force depends on normal deformation and its rate, and no explicit relationship with the orientation is considered in Eq. (5),

$$
F_{i j}^{n}(\xi, \dot{\xi})=\frac{4 E_{i j}^{*} \sqrt{R_{i j}^{*}}}{3}\left(\xi_{i j}^{3 / 2}+\eta_{i j} \sqrt{\xi_{i j}} \dot{\xi}_{i j}\right)
$$

Here, $\xi_{i j}$ is the total normal deformation of collided particles $i$ and $j$ at their contact point. $R_{i j}^{*}$ and $E_{i j}^{*}$ are the equivalent radius and Young's modulus, respectively, which are defined as follows,

$$
\frac{1}{E_{i j}^{*}}=\frac{1-v_{i}^{2}}{E_{i}}+\frac{1-v_{j}^{2}}{E_{j}}
$$




$$
\frac{1}{R_{i j}^{*}}=\frac{1}{R_{i}}+\frac{1}{R_{j}}
$$

Furthermore, $\eta_{i j}$ is the internal viscosity related to the restitution coefficient, $\in_{i j}$, via the following Eq. (7) [30, 31],

$$
\epsilon_{i j}=\exp \left(-\frac{\pi \eta_{i j}}{\sqrt{2 m_{i j}^{*} K_{i j}^{n}-\eta_{i j}^{2}}}\right)
$$

Here, $m_{i j}^{*}$ is the equivalent mass of the interacting particles $i$ and $j$, and $K_{i j}^{n}$ is the normal equivalent spring between these particles,

$$
\begin{gathered}
\frac{1}{m_{i j}^{*}}=\frac{1}{m_{i}}+\frac{1}{m_{j}} \\
K_{i j}^{n}=\frac{4}{3} E_{i j}^{*} \sqrt{R_{i j}^{*}}
\end{gathered}
$$

Furthermore, the tangential interaction force between two bodies is obtained as follows,

$$
F_{i j}^{t}=-\operatorname{sign}\left(v_{\mathrm{rel}}^{t}\right) \min \left(\gamma_{i j}^{t}\left|v_{i j}^{t}\right|, \mu_{i j}\left|F_{i j}^{n}\right|\right)
$$

where $v_{i j}^{t}, \gamma_{i j}^{t}$, and $\mu_{i j}$ are the relative tangential velocity and the viscous and dry friction coefficients between the interacting bodies, respectively. The governing equations in the system are time-dependent, and numerical integration with respect to time is performed in process of obtaining the numerical solution. Here, the Gear integration method [32] is employed for this purpose, and sensitivity analysis is performed to ensure adequate accuracy.

To estimate the wear using the DEM, the Finnie model was implemented [33]. For this purpose, each surface was meshed into small triangular elements, and the wear of each element is evaluated as a summation of the wear due to the impact of all the particles on that element. During the process of solving the DEM, the position and velocity of all the particles and surface elements are known at each time step. Therefore, the following equation can be employed to evaluate the wear due to each impact at any time step $[9,33]$ :

$$
W=K_{\mathrm{F}} m v^{2} Z(\alpha)
$$

where $W$ is the removed volume from a point due to the impact of a particle with mass $m$, speed $v$, and impact angle $\alpha$ (Fig. 1). $K_{\mathrm{F}}$ is the Finnie wear constant, and $Z(\alpha)$ represents the dependency on the impact angle, according to the following Eqs. (12a) and (12b) $[9,33]$,

$$
\begin{gathered}
Z(\alpha)=\frac{1}{k}\left(\sin (2 \alpha)-\frac{6}{k} \sin ^{2}(\alpha)\right), \text { if } \tan \alpha \leq \frac{k}{6} \\
Z(\alpha)=\frac{1}{6} \cos ^{2}(\alpha), \text { if } \tan \alpha \geq \frac{k}{6}
\end{gathered}
$$

Here, $k$ is a conditional constant depending on the material characteristics and is related to the impact angle that causes the maximum wear. A few experimental tests are needed to determine the constants $k$ and $K_{\mathrm{F}}$ in Eqs. (11) and (12). The experimental data should match the mathematical model. However, in theoretical studies, including the numerical simulations of the present study, values are assigned to these constants. In this regard, assumed values and relevant explanations are presented in Section 3. In the DEM, the removed volume is divided by the affected surface to obtain the depth of the wear, and the unit of the wear becomes the depth of the wear. To clarify the process of wear estimation via the DEM, the contact time can be divided into the penetration and repulsion periods. During each of these two periods, the magnitude and direction of the particle velocity change gradually. Thus, the total wear is determined via integration of the wear rate over the contact time. For this purpose, the wear rate is calculated during each impact by using the following Eq. (13),

$$
w=\frac{\mathrm{d} W}{\mathrm{~d} t}=\frac{\partial W}{\partial v} \frac{\partial v}{\partial t}+\frac{\partial W}{\partial \alpha} \frac{\partial \alpha}{\partial t}
$$

It is a logical assumption that during the penetration

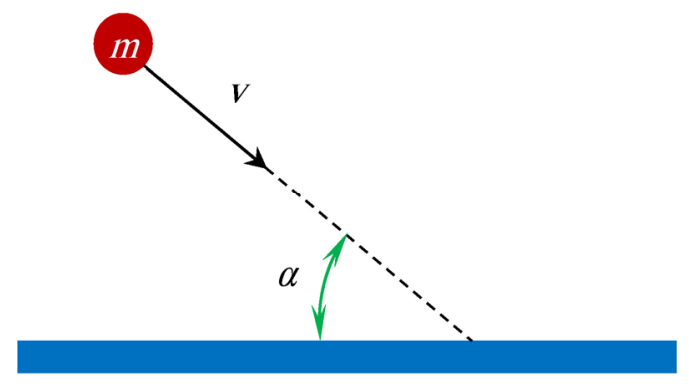

Fig. 1 Impact of a particle on a surface element. 
period, the particle transfers its energy to the surface, causing wear, and negligible wear occurs during the repulsion. Therefore, integration of the wear rate over the penetration period determines the wear. On the other hand, the direction of the particle velocity is assumed to remain almost unchanged during the penetration period; thus, the last term in Eq. (13) can be neglected. Hence, by substituting Eq. (11) into Eq. (13) and employing the well-known equilibrium equation $\mathrm{d} V / \mathrm{d} t=F / m$, the following Eq. (14) is obtained,

$$
w \approx \frac{\partial W}{\partial v} \frac{\partial v}{\partial t}=2 K_{\mathrm{F}} Z(\alpha) v F
$$

Here, $F$ is the contact force. Finally, the wear due to a collision is obtained via integration of Eq. (14) over the penetration time, as follows,

$$
W=\int_{0}^{t_{\mathrm{p}}} w \mathrm{~d} t=2 K_{\mathrm{F}} \int_{0}^{t_{\mathrm{p}}} H(\vec{v}, \vec{q}) Z(\alpha) v F \mathrm{~d} t
$$

Here, $H(\vec{v}, \vec{q})$ is the Heaviside function defined below, which applies the rule that the integration kernel is non-zero only during the penetration period,

$$
H(\vec{v}, \vec{q})=\left\{\begin{array}{l}
1, \text { if } \vec{v} \cdot \vec{q} \geq 0 \\
0, \text { if } \vec{v} \cdot \vec{q}<0
\end{array}\right\}
$$

Here, $\vec{q}$ is a unit vector pointing from the particle center to the contact point.

To conduct the simulations, adequate codes were developed by using the open-source DEM code LIGGGHTS [33], which is a special version of LAMMPS for modeling granular media. A set of parallel computers with a total of 24 cores and a Linux operation system was used to run the simulations. Graphical results were obtained using ParaView [34].

\section{Numerical results for single-particle model}

As mentioned previously, the Finnie model is employed here to evaluate the erosive wear. According to this model, the particle mass, impact angle, and Finnie coefficient participate in the mathematical formulation implicitly. However, in addition to these parameters, others, including the particle size, surface mesh size,
Young's modulus, Poisson's ratio, and restitution coefficient, are studied using a single-particle model. In this part, a spherical particle is dropped at the center of an equilateral triangular surface. The orientation of the surface is changed to provide different impact angles.

Table 1 presents the ranges of the parameters investigated in this part. The effect of each parameter is studied individually, meaning that the others are fixed at the reference values presented in Table 1. In the reported results, including the graphs, when the value of any parameter is not given, the reference value should be assumed.

As mentioned previously, the accuracy and convergence of the results depend on the time step. Therefore, sensitivity analysis is performed to obtain a proper time step leading to reliable results. For this purpose, several simulations are conducted by selecting different values for the time step in the range of 0.01-1.84 $\mu \mathrm{s}$, and the results are plotted in Fig. 2. To provide a clear indication of the sensitivity to the time step, the results in this figure are normalized by dividing them by the smallest one. The dependence of the wear on the time step disappears when the time step is reduced. When the time step is $<0.075 \mu \mathrm{s}$, the graph almost becomes plateau, which means that acceptable accuracy is obtained regardless of the time step.

\begin{tabular}{|c|c|}
\hline Parameter & Range \\
\hline Particle diameter (mm) & $\begin{array}{c}0.0002,0.001, \mathbf{0 . 0 0 2}, 0.008,0.016 \\
0.03,0.04\end{array}$ \\
\hline Impact velocity $(V)(\mathrm{m} / \mathrm{s})$ & $\begin{array}{c}\mathbf{2 . 2 3}, 2.35,2.91,3.22,3.52,4.04 \\
5.65,7.74,9.96\end{array}$ \\
\hline Impact angle $\left(\alpha^{\circ}\right)$ & $0, \mathbf{1 0}, 20,30,40,50,60,70,80,90$ \\
\hline Surface area $(A)\left(\mathrm{mm}^{2}\right)$ & $\mathbf{1 0 . 8 2 5}, 43.301,97.428,173.205$ \\
\hline Particle density $(\rho)\left(\mathrm{kg} / \mathrm{m}^{3}\right)$ & $500,1500, \mathbf{2 5 1 6}, 3500,5000$ \\
\hline $\begin{array}{l}\text { Finnie coefficient }\left(K_{\mathrm{F}}\right) \\
\qquad\left(\mathrm{Pa}^{-1}\right)\end{array}$ & $0.6,0.75,1.11, \mathbf{1 . 3 3}, 2,4$ \\
\hline Restitution coefficient $(\in)$ & $0.1,0.2,0.4, \mathbf{0 . 5}, 0.6,0.8,1$ \\
\hline \multirow{2}{*}{$\begin{array}{l}\text { Young's modulus } \\
(\mathrm{MPa})\end{array}$} & 41 \\
\hline & $5,15, \mathbf{2 5}, 30,45$ \\
\hline \multirow{2}{*}{ Poisson's ratio $(v)$} & 0.22 \\
\hline & $0.10,0.15, \mathbf{0 . 2 5}, 0.3$ \\
\hline
\end{tabular}

Table 1 Range of parameters in the single-particle model (reference values are presented in bold). 


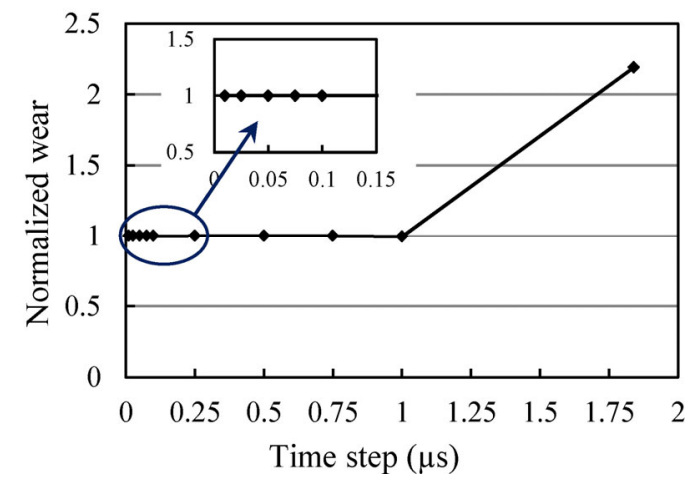

Fig. 2 Sensitivity analysis versus the time step.

\subsection{Effect of surface mesh size}

In the implementation of the DEM, each surface must be meshed into small triangular elements via discretization. To reveal the effects of the meshing on the results, a spherical particle is dropped at the center of an equilateral triangular surface, as shown in Fig. 3. The same problem is simulated under three different meshing cases consisting of 1,4 , and 16 elements. By dividing the data by the smallest one, the normalized wear results are obtained. As shown in Fig. 3, the wear values of the affected element are

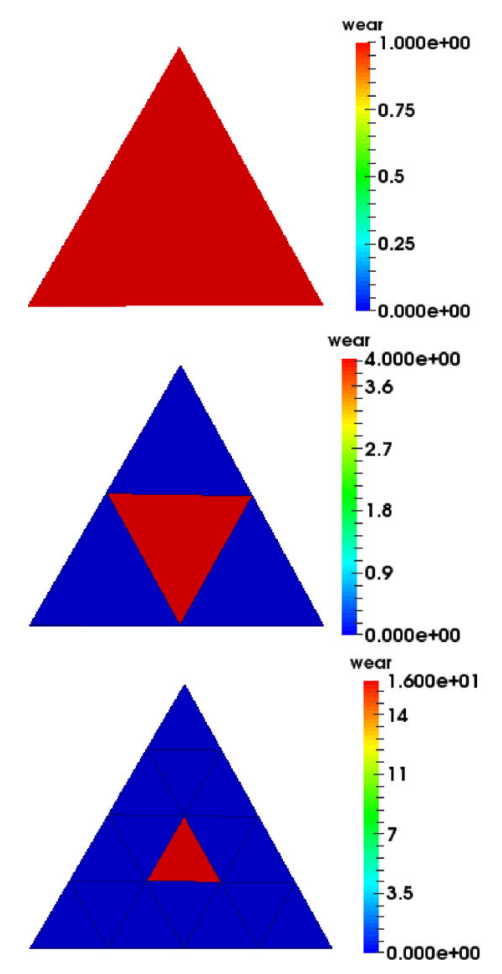

Fig. 3 Effect of the meshing on the evaluated wear in the DEM (the impact angle is $20^{\circ}$, and the other parameters are set as the reference values; the values represent the normalized wear).
1,4 , and 16 in the cases where 1,4 , and 16 elements are used, respectively. In the case where 16 elements are used, the evaluated wear is 4 and 16 times larger than those for meshing with 4 elements and 1 element, respectively. Moreover, the wear occurs only in the element that is impacted by the particle. Thus, the particle transfers its kinetic energy to only the area of the impacted element, rather than the entire area of the surface. However, multiplying the evaluated wear by the area of the affected element gives an identical value for all three meshing cases. Hence, the reported value for the wear is the depth of the wear of the affected element. It can be concluded that a particle impact causes a certain volume to be removed from the target body; however, a smaller affected element yields greater depth of the local wear. That is, the local wear depends on the meshing, such that refinement of the meshing leads to the evaluation of more localized wear. Nevertheless, as explained previously, the overall wear of the entire surface is identical for all meshing numbers.

\subsection{Effect of impact angle}

In the aforementioned Finnie analytical model, the wear depends on the impact angle according to certain functions. However, there is the question of how the DEM code captures this model and the question of their coincidence. As explained in the previous section, the constant $k$ in the Finnie model is a material characteristic; however, as an example case, we assumed that the maximum wear occurs at the impact angle $\alpha_{\mathrm{c}}=38^{\circ}$, leading to $k=12$. To compare the simulation results with the analytical Finnie model, several simulations were conducted with different values of the impact angle, while the other parameters were fixed at the reference values. The normalized wear (obtained by dividing by the largest value) versus the impact angle is plotted in Fig. 4. According to these graphs, in most ranges of the impact angle, both approaches are in good agreement; however, a deviation between the DEM and the analytical Finnie model is observed. The experimental results indicate that the impact angle that causes the largest wear depends on the material behavior; for ductile materials, this angle is in the range of $20^{\circ}-60^{\circ}$, whereas for brittle materials, it is approximately $90^{\circ}[13,35]$. Therefore, the current 


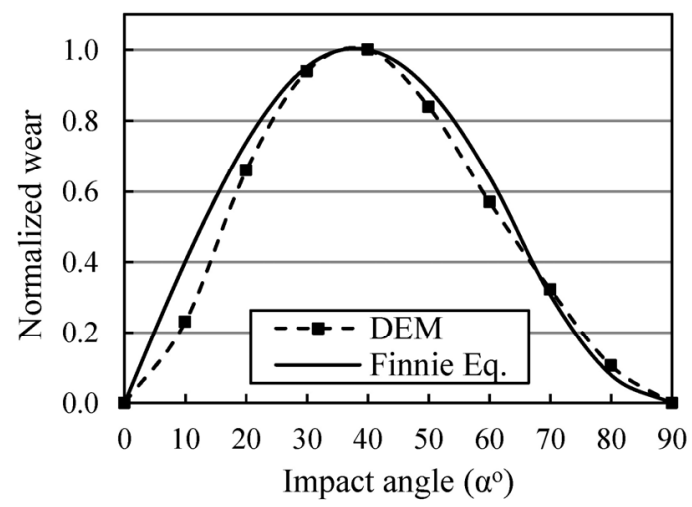

Fig. 4 Dependence of the wear on the impact angle of a single particle, obtained using the DEM and Finnie equation.

model is suitable for ductile materials. The deviations between the analytical and numerical results can be due to differences between the implementation of the wear model in the DEM and the analytical approach. In the analytical model, the particle speed at the beginning of the collision is considered, whereas in the DEM, the more complicated scenario illustrated in Section 2.1 is implemented.

\subsection{Effects of impact velocity}

To examine the effect of the impact speed on the wear, simulations were conducted for different values of the impact speed in the range of $2-10 \mathrm{~m} / \mathrm{s}$. To properly indicate the dependency, the results were divided by the largest value, and the obtained normalized results are plotted in Fig. 5. As shown in Fig. 5, the DEM results imply that the dependency of the wear on the speed has the form of a power function, such as $v^{1.87}$. The same trend in the form of $v^{n}$ was observed for other simulation conditions, and the value of the power

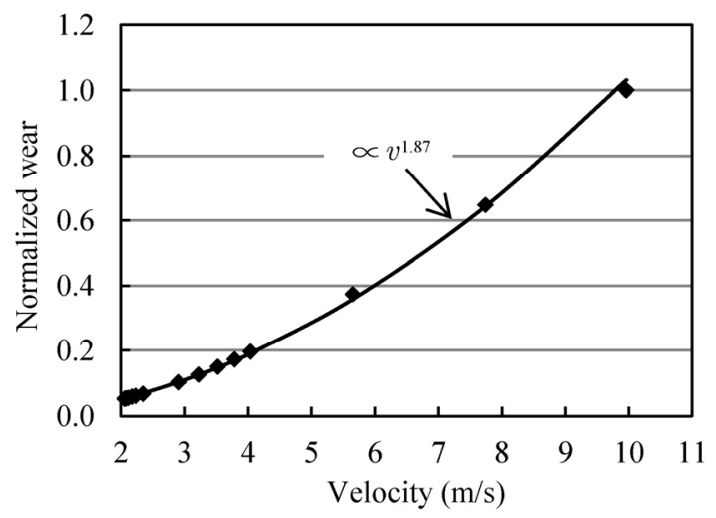

Fig. 5 Variations of the wear versus the particle velocity $\left(\alpha=10^{\circ}\right.$ and the other variables are at the reference values). factor, $n$, depends on the impact angle. This agrees with previous experimental results, such as those reported in Ref. [36]. Further discussion regarding this matter is omitted here, and adequate explanations will be presented in the next section.

\subsection{Effect of particle size}

According to previously reported experimental studies, increasing the particle size leads to a higher wear [5, 37-39]. Furthermore, it has been proven that there is no unique dependency for all ranges of the particle size [40, 41]. No consensus exists in the literature regarding the mathematical model for the dependence of the wear on the particle size. For instance, a polynomial function was presented in [42], and a power function was proposed in Refs. [43-45]. In the present study, for different values of the particle diameter in the range of $0.2-40 \mathrm{~mm}$, DEM simulations were conducted, and the normalized results are shown in Fig. 6. Normalization was performed by dividing the results by the largest one. In Fig. 6, a fitted curve for the DEM results is also plotted. The wear is related to the

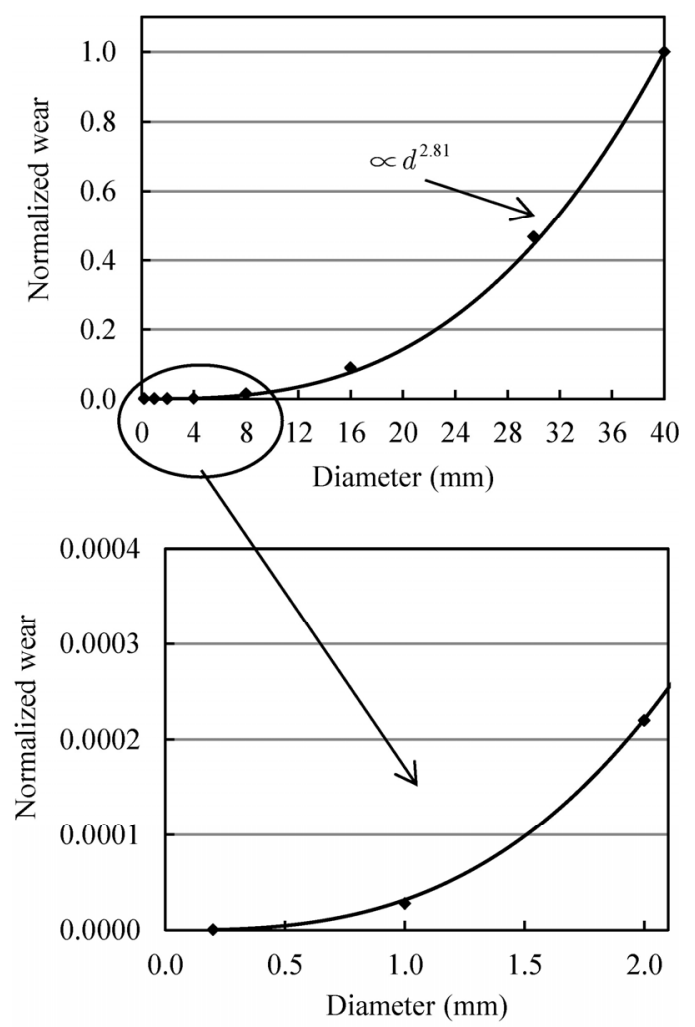

Fig. 6 Variation of the wear versus the diameter of the impacting particle ( $\alpha=40^{\circ}$ and the other variables are at the reference values). 
particle diameter almost in the form of $d^{2.81}$. Thus, for the range of particles studied, the power function is suggested for the dependence of the wear on the particle diameter.

\subsection{Effect of particle density}

According to the Finnie model, the wear is linearly related to the particle mass; however, the DEM model was verified via simulation of several problems with different particle densities in range of $500-5,000 \mathrm{~kg} / \mathrm{s}$. The normalized results depicted in Fig. 7 show that the wear has a linear relationship with the particle density. This result demonstrates the idea that the wear of a surface is due to the kinetic energy of the impinging particle. The kinetic energy varies linearly with respect to the particle mass; thus, in the case where the particle volume is constant, this energy has a linear relationship with the particle density. However, other wear estimator models have been proposed for brittle materials that suggest a nonlinear dependence of the wear on the particle density [46, 47]. The present results are suitable for ductile materials.

\subsection{Effects of restitution coefficient, Young's modulus, and Poisson's ratio}

According to the Finnie model, there is no implicit relationship between the wear and the restitution coefficient, Young's modulus, and Poisson's ratio. However, the wear depends on the velocity and the impact force, which is related to these characteristics. Thus, several simulations were conducted with different values for each of the introduced properties. Here, the

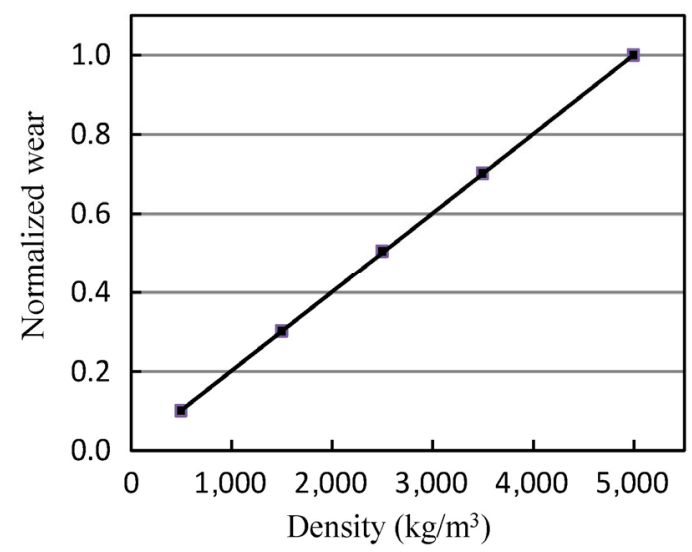

Fig. 7 Variations of the wear versus the particle density ( $\alpha=30^{\circ}$ and the other variables are at the reference values). impact angle was $50^{\circ}$, and the other characteristics were fixed at their reference values. For the ranges of the introduced characteristics, various wear results were obtained. They were normalized by dividing them by the largest value for each case study and are plotted in Figs. 8-10. As shown in Fig. 8, the wear is

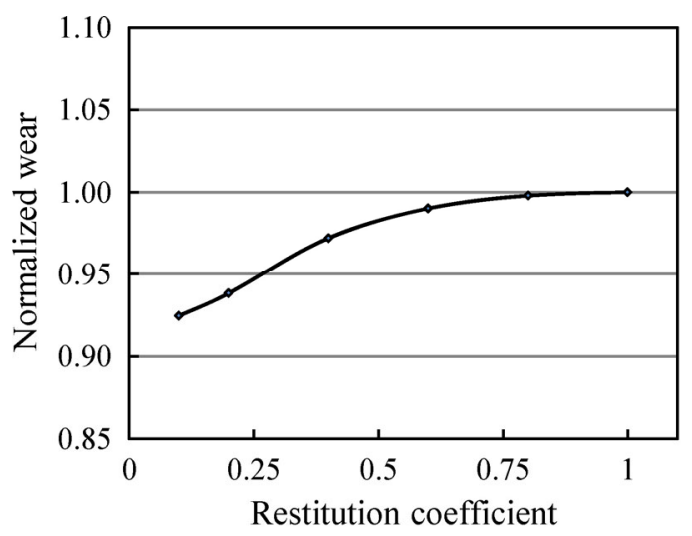

Fig. 8 Variation of the wear versus the restitution coefficient ( $\alpha=50^{\circ}$ and the other variables are at the reference values).

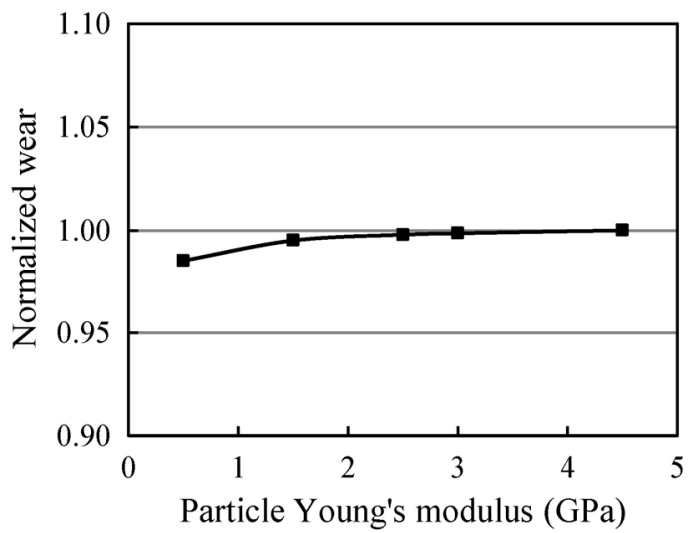

Fig. 9 Variations of the wear versus the surface Young's modulus ( $\alpha=10^{\circ}$ and the other variables are at the reference values).

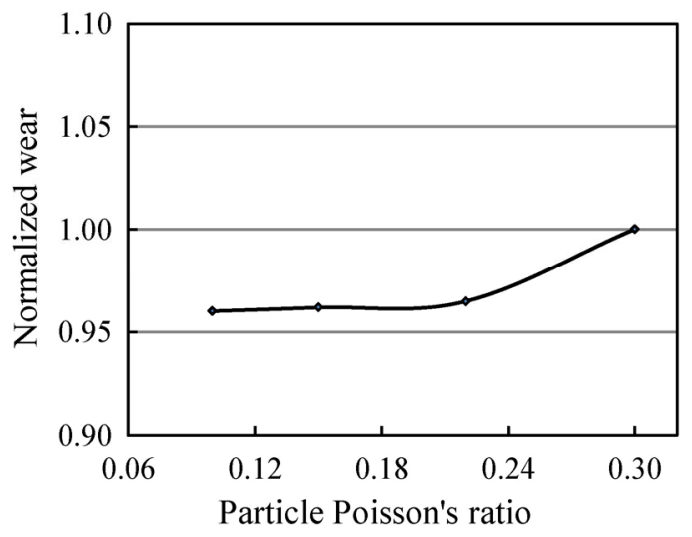

Fig. 10 Variations of the wear versus the particle Poisson's ratio ( $\alpha=10^{\circ}$ and the other variables are at the reference values). 
not significantly related to the restitution coefficient; with the increase of this coefficient, the wear increases slightly. This dependency can be due to the fact that by increasing the restitution coefficient, the dissipated energy is decreased, and the higher remaining kinetic energy can contribute to the increase of the wear. On the other hand, the collision time varies with respect to the restitution coefficient [48] and can affect the integration domain introduced in Eq. (15). This can be another illustration of the dependency of the wear on the restitution coefficient; however, it is not a substantial relationship.

Regarding the dependency of the wear on the elastic constants - the Young's modulus and Poisson's ratio-the results are plotted in Figs. 9 and 10. Similar to the case of the dependency on the restitution coefficient, small variations are observed in these graphs. In the Finnie model, there is no apparent relationship between the wear and these constants. Nevertheless, owing to Eqs. (5) and (9) and as proved in [48], these parameters participate in the overall elastic stiffness of the contact zone and thus affect the collision time and interacting force. Therefore, weak relationships between the wear and elastic constants are expected, which agrees with the findings in Ref. [49]. The graphs show that with the increase of the surface Young's modulus and Poisson's ratio, the wear is increased slightly. To discuss such dependencies, Eqs. (5) and (9) should be reloaded as indicate that by increasing these properties, the contact region becomes stiffer leading to a creation a higher energy for making the wear.

\section{Flow erosion}

Up to now, the wear was studied using the singleparticle model to reveal the basic correlations between the wear and influencing parameters. As described in this section, another set of simulations was conducted to study the erosion of a surface under a flow of particles. The flow erosion was studied using the model depicted in Fig. 11, in which two bodies rotate around a fixed axis inside a container filled with spherical particles. This model is similar but not identical to the experimental rigs reported in Refs. [5, 45, 50].

The physical characteristics of the particles and fixed parameters are presented in Table 2. To examine the effects of the sample orientation and speed on the wear, different values were assigned to these parameters in the ranges shown in Table 3. To clarify the details of the model and the definition of the surface orientation (angle), different parts and a schematic diagram were drawn, as presented in Fig. 12. As shown, the surface angle $\beta$, is the angle between the surface and the circular path in the container.

As an example, a contour plot of the flow erosion for a typical case is presented in Fig. 13. It is observed that the wear on each sample surface is not uniform. As proved previously, the impact direction is an important parameter affecting the wear and can cause

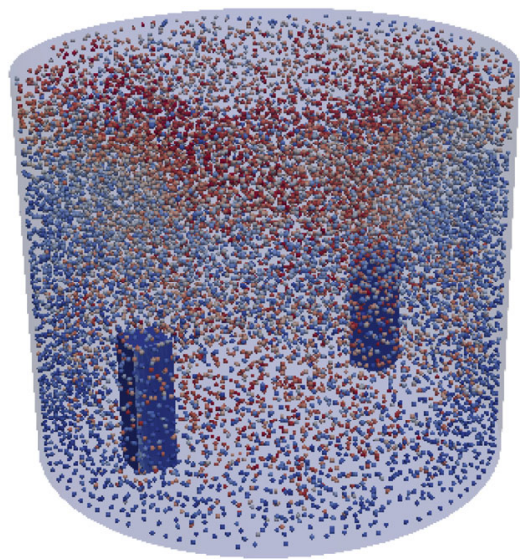

Fig. 11 Simulation of the flow erosion using the DEM.

Table 2 Physical characteristics of the particles and other fixed factors in the flow-erosion simulations.

\begin{tabular}{llc}
\hline \multicolumn{1}{c}{ Property } & & Value \\
\hline Young's modulus $(\mathrm{MPa})$ & particles & 41 \\
& surfaces & 25 \\
Finnie Coefficient $\left(\mathrm{GPa}^{-1}\right)$ & & $2.86 \mathrm{E}-11$ \\
Poisson's ratio & particles & 0.22 \\
Friction coefficient & surfaces & 0.25 \\
Restitution coefficient & & 0.3 \\
Particles density $\left(\mathrm{kg} / \mathrm{m}^{3}\right)$ & & 0.5 \\
Particles diameter $(\mathrm{mm})$ & & 2516 \\
Number of particles & & 0.6 \\
Simulation time $(\mathrm{s})$ & & 17232 \\
\hline
\end{tabular}

Table 3 Range of variables for studying the flow erosion.

\begin{tabular}{cc}
\hline Parameter & Values \\
\hline Sample speed $(\mathrm{m} / \mathrm{s})$ & $1.5,3,4.5,6$ \\
Sample angle, $\beta\left(^{\circ}\right)$ & $0,15,45,75,90$ \\
\hline
\end{tabular}




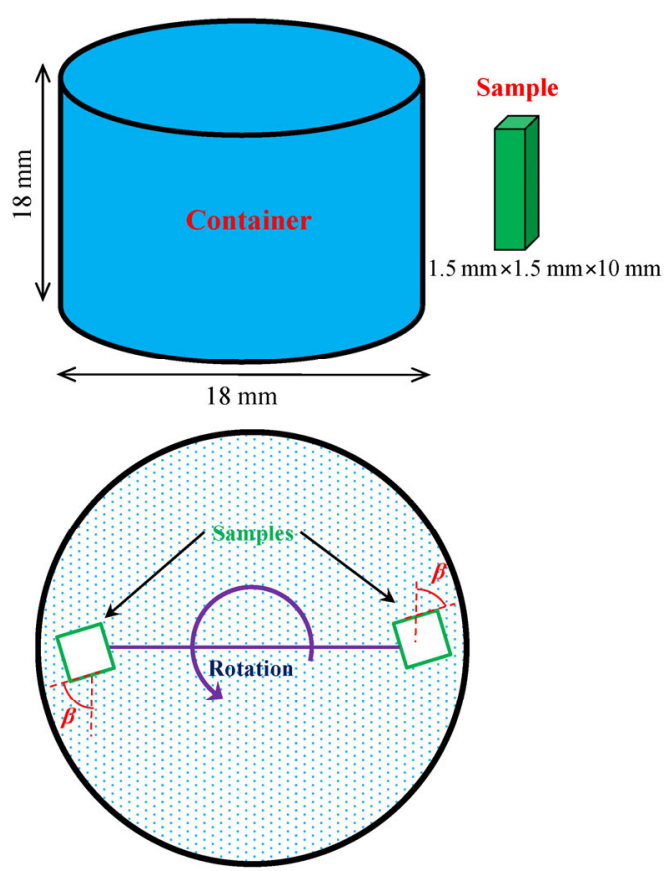

Fig. 12 Container, samples, and section view for the flow-erosion simulations.

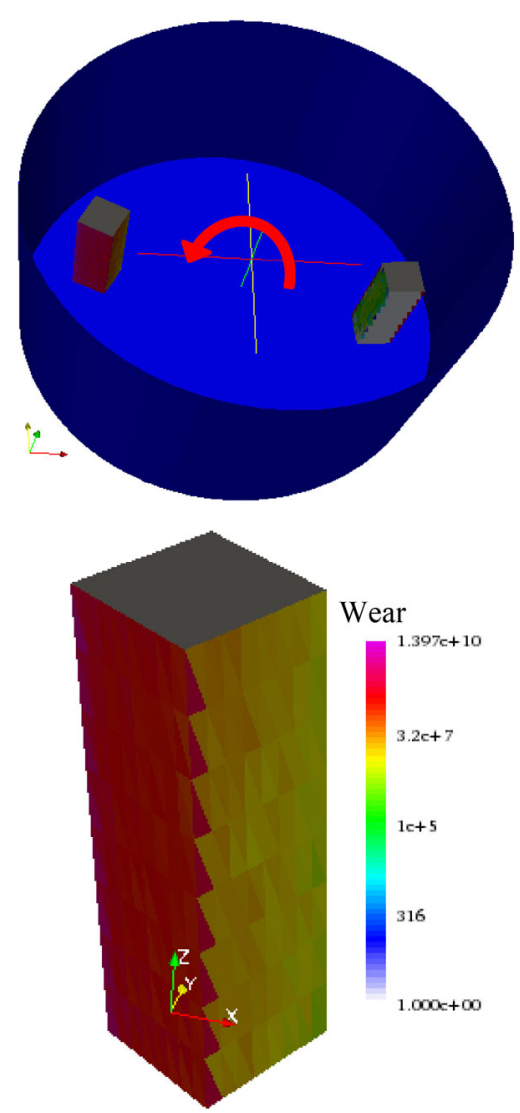

Fig. 13 Location of the samples inside the container filled with particles and contours of the erosive wear on the sample surfaces. ( $d=0.6 \mathrm{~mm}$ and two surface orientations are $\beta=80^{\circ}$ and $\beta=60^{\circ}$; The particles are hidden.) such wear regimes. Each surface of the sample is meshed with 128 elements, each of which experiences its own flow state, leading to deviation of the local wear between different points on the surface. To facilitate comparison and numerical study, the total erosion of all the elements for each flat surface was determined as the overall erosive wear of this surface, as presented in the following subsections.

\subsection{Erosion versus time}

Variations of the erosion versus time for different speeds and different sample angles are plotted in Fig. 14. The distance can be determined when the rotation speed, radius of rotation, and elapsed time are known. For all speeds and all angles, the wear varies linearly with respect to time (distance). This trend agrees with experimental results obtained for a steel material [51]. However, zooming in on the initial steps presented in Fig. 15 indicates that initial regions of the graphs are nonlinear. This can be due to the fact that the system started in a static condition and took some time to reach steady-state conditions. Thus, the wear varies non-linearly in transient conditions; however, it gradually approaches a linear trend.

\subsection{Erosion versus sample angle}

The experimental studies proved that the angle that causes maximum wear depends on the material behavior. For ductile materials, the maximum wear occurs at small angles in the range of $20^{\circ}-60^{\circ}$, whereas for brittle materials, it occurs around $90^{\circ}$ [13]. In the present study, the role of the sample angle, which is introduced in Fig. 12, is studied by adjusting the angle $\beta$. For a constant traveled distance (14.72 revolutions $=0.000555 \mathrm{~m}$ ), the graphs of the wear versus the surface orientation are plotted in Fig. 16. For all speeds of the surface inside the particulate medium, the maximum wear occurs at $\beta=45^{\circ}$. Therefore, it can be concluded that the constants assigned to the employed Finnie model are suitable for ductile materials. At angles of $0^{\circ}$ and $90^{\circ}$, the wear is too small. This can be due to the fact that when the angle is $0^{\circ}$, the particles slip on the surface, and when the angle is $90^{\circ}$, the particles penetrate the surface and return without considerable wear. The simulation conditions used in this study are similar to the 

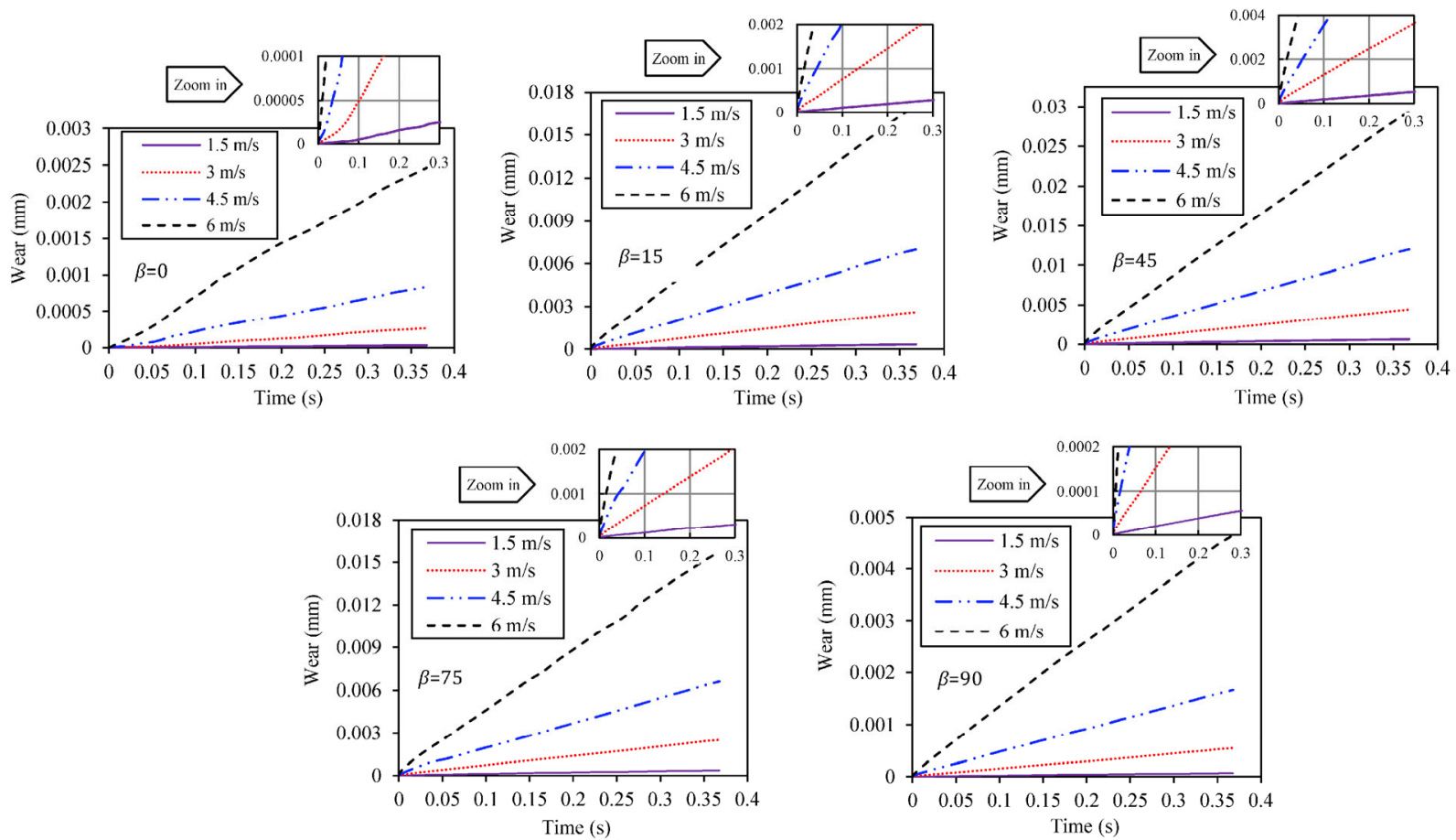

Fig. 14 Variation of the erosive wear versus time (distance) for different values of the speed and sample angle (particle diameter is $0.6 \mathrm{~mm})$. Note: the vertical axes are not in the same range.

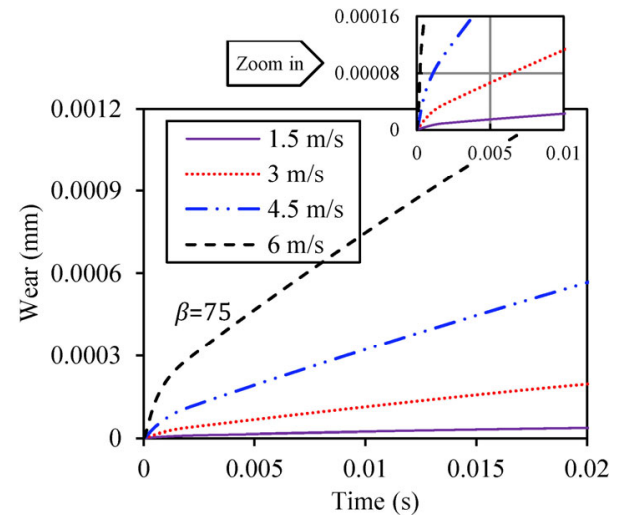

Fig. 15 Magnification of the erosion graphs for the initial transient conditions.

experimental setup in Ref. [45]. Although the numerical results cannot be compared directly, the trends of the dependency of the wear on the sample orientation in this study are similar to those reported in Ref. [45]. Moreover, we observed that the maximum wear occurred for the sample orientation of $\beta=45^{\circ}$, which is in agreement with the experimental results in Ref. [45].

\subsection{Erosion versus speed}

In the previous section, the effects of the particle speed

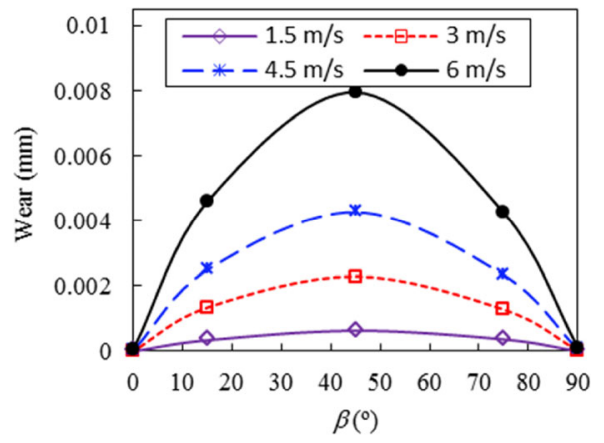

Fig. 16 Variation of the flow erosion versus the angle of the surface inside the flow, at different speeds.

on the wear were studied using a single-particle model. It was proven that the wear is related to the particle speed via a power function; however, there is a question about this correlation when the sample is under the impact of a particle flow. To answer this question for more general conditions, several problems were simulated for different values of the sample orientation and various speeds. For different sample angles, graphs of the erosion versus the speed are presented in Fig. 17. For identical elapsed time, different speeds lead to different traveled distances; thus, these graphs are plotted for an identical traveled distance. The values 
of the wear change with respect to the sample angle; however, similar trends are observed with regard to the speed dependency. Furthermore, we attempted to extract a function to represent the speed dependency, as shown in Fig. 18, for a few cases and found that at

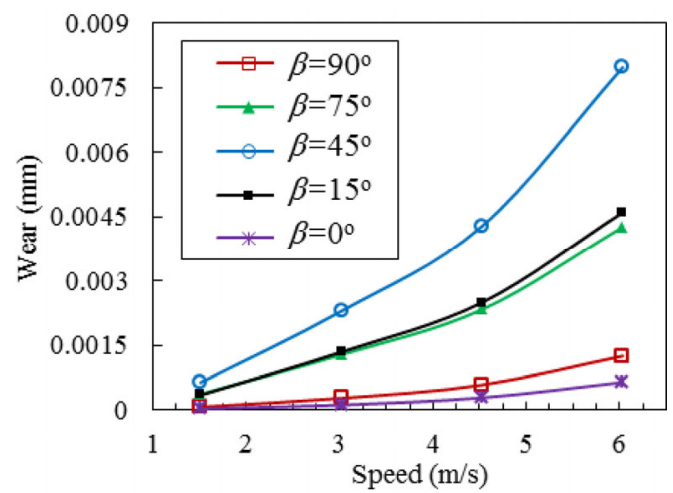

Fig. 17 Erosion versus the speed of the surface inside the particulate medium (the other parameters are at their reference values).
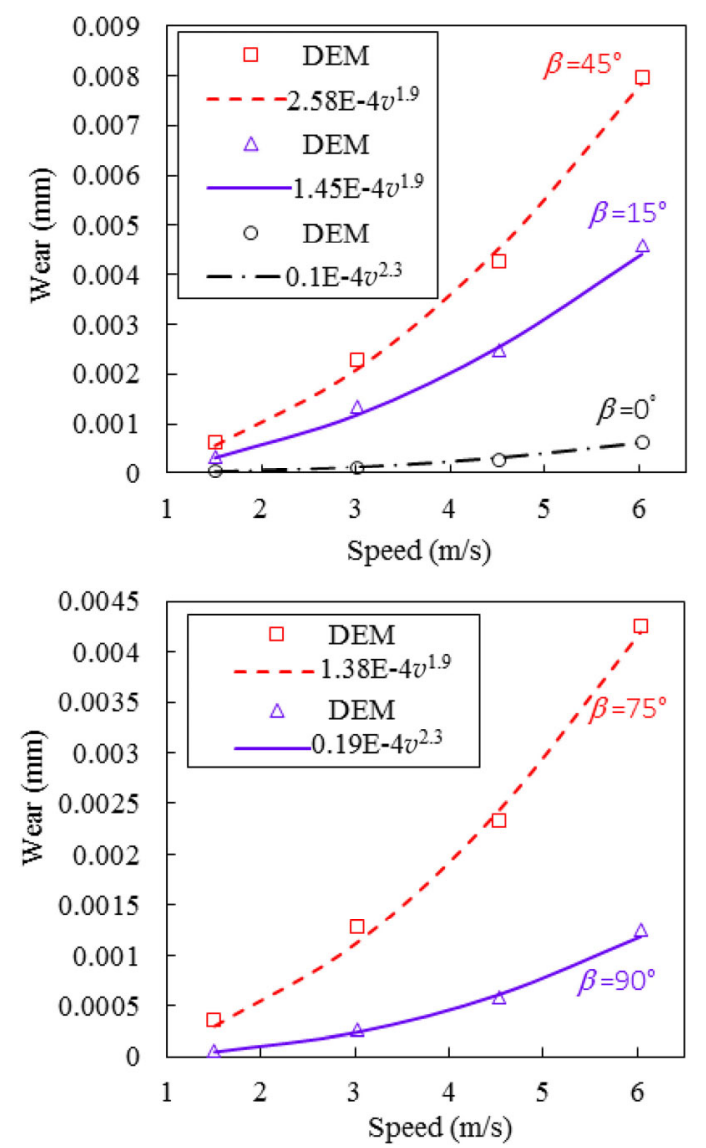

Fig. 18 Variation of the erosion versus the speed of the samples inside the particulate medium, along with the fitted power function (particle radius is $0.3 \mathrm{~mm}$, and the other parameters are at their reference values). all sample angles, the erosion depends on the speed via a power function $C v^{n}$. Interestingly, these graphs are similar to the one previously proposed for the single-particle model. Regarding the correlation function, agreement is observed between this form and the results in the literature, especially the experimental studies in Refs. [11, 12, 36, 52-54]. In fact, the constant $C$ and the power $n$ depend on the working conditions and are obtained by fitting experimental/numerical data to the power function. For instance, values in the range of 1.5-3.5 have been reported for the power, $n$ [11, 12, 36, 52-54]. These constants were quantified via interpolation of the DEM results, and their values for different sample angles are presented in Table 4. The power constant, $n$, is approximately 2.3 for angles of $\beta=0^{\circ}$ and $\beta=90^{\circ}$ and approximately 1.9 for the other angles.

Table 4 Constant and the power in the function of the erosion versus the speed.

\begin{tabular}{ccc}
\hline Sample angle $(\beta)$ & $C$ & $n$ \\
\hline 0 & $0.1 \mathrm{E}-4$ & 2.3 \\
15 & $1.45 \mathrm{E}-4$ & 1.9 \\
45 & $2.58 \mathrm{E}-4$ & 1.9 \\
75 & $1.38 \mathrm{E}-4$ & 1.9 \\
90 & $0.19 \mathrm{E}-5$ & 2.3 \\
\hline
\end{tabular}

\section{Conclusions}

The DEM was employed successfully to study the surface wear under the impact of granular materials. First, a single-particle model was used to calibrate the wear parameters and identify the basic factors that affect the wear process. It was proven that the impact angle, impact speed, particle size, and particle density significantly affect the wear. Furthermore, it was observed that physical characteristics - the Young's modulus, Poisson's ratio, and restitution coefficientaffect the wear to some extent. As a more general case, simulations were conducted to identify the parameters influencing the surface erosion under the flow of particles. The results showed that in steady-state conditions, the flow erosion increases linearly with respect to time. The orientation of the surface inside the particulate medium is a key factor affecting the wear. It was observed that the erosion is related to the speed 
via a power function; however, the function coefficients depend on the angle of the surface. The trends of a few dependences were in good agreement with the relevant experimental data in the literature. DEM simulations allow the observation and comparison of the qualitative relationships between the wear and influential parameters.

\section{Acknowledgements}

The work was financially supported by the Iran National Science Foundation (INSF) under Grant No. 93038047.

Open Access: The articles published in this journal are distributed under the terms of the Creative Commons Attribution 4.0 International License (http:// creativecommons.org/licenses/by/4.0/), which permits unrestricted use, distribution, and reproduction in any medium, provided you give appropriate credit to the original author(s) and the source, provide a link to the Creative Commons license, and indicate if changes were made.

\section{References}

[1] Holmberg K, Erdemir A. Influence of tribology on global energy consumption, costs and emissions. Friction, 5: 263284 (2017)

[2] Burwell J T. Survey of possible wear mechanisms. Wear, 1: 119-141 (1957)

[3] Varenberg M. Towards a unified classification of wear. Friction, 1: 333-340 (2013)

[4] Archard J F. A crossed-cylinders friction machine. Wear, 2: 21-27 (1958)

[5] Lynn R S, Wong K K, Clark H M. On the particle size effect in slurry erosion. Wear, 149: 55-71 (1991)

[6] Chacon-Nava J G, Martinez-Villafañe A, Almeraya-Calderon F, Cabral-Miramontes J A, Stack M M. Some remarks on particle size effects on the abrasion of a range of Fe based alloys. Tribology International, 43: 1307-1317 (2010)

[7] Ojala N, Valtonen K, Antikainen A, Kemppainen A, Minkkinen J, Oja O, Kuokkala V-T. Wear performance of quenched wear resistant steels in abrasive slurry erosion. Wear, 354-355: 21-31 (2016)

[8] Woldman M, van der Heide E, Schipper D J, Tinga T, Masen M A. Investigating the influence of sand particle properties on abrasive wear behaviour. Wear, 294-295: 419-426 (2012)
[9] Finnie I. Erosion of surfaces by solid particles. Wear, 3: 87-103 (1960)

[10] Archard J. Contact, rubbing of flat surfaces. Journal of applied physics, 24: 981-988 (1953)

[11] Wellinger K, Breckel H. Kenngrössen und Verschleiss beim Stoss metallischer Werkstoffe. Wear, 13: 257-281 (1969)

[12] Hutchings I M. Tribology: friction and wear of engineering materials. United Kingdom: Elsevier 1992.

[13] Rabinowicz E. Friction and wear of materials, 2 ed. New York: John Wiley and Sons, 1995.

[14] Xue Y, Chen J, Guo S, Meng Q, Luo J. Finite element simulation and experimental test of the wear behavior for self-lubricating spherical plain bearings. Friction: (2018)

[15] Zhu A, He D, He S, Luo W. Material removal mechanism of copper chemical mechanical polishing with different particle sizes based on quasi-continuum method. Friction, 5: 99-107 (2017)

[16] Abdullah O I, Schlattmann J. Thermal behavior of friction clutch disc based on uniform pressure and uniform wear assumptions. Friction, 4: 228-237 (2016)

[17] Liu H, Liu H, Zhu C, Wei P, Tang J. Tribological behavior of coated spur gear pairs with tooth surface roughness. Friction: (2018)

[18] Cleary P W. Predicting charge motion, power draw, segregation and wear in ball mills using discrete element methods. Minerals Engineering, 11: 1061-1080 (1998)

[19] Kalala J T, Moys M H. Discrete element method modelling of liner wear in dry ball milling. Journal of the Southern African Institute of Mining and Metallurgy, 104(10): 597-602 (2004)

[20] Kalala J T, Bwalya M, Moys M H. Discrete element method (DEM) modelling of evolving mill liner profiles due to wear. Part II. Industrial case study. Minerals Engineering, 18: 1392-1397 (2005)

[21] Ashrafizadeh H, Ashrafizadeh F. A numerical 3D simulation for prediction of wear caused by solid particle impact. Wear, 276-277: 75-84 (2012)

[22] Zhang H, Tan Y, Yang D, Trias F X, Jiang S, Sheng Y, Oliva A. Numerical investigation of the location of maximum erosive wear damage in elbow: Effect of slurry velocity, bend orientation and angle of elbow. Powder Technology, 217: 467-476 (2012)

[23] Tan Y, Zhang H, Yang D, Jiang S, Song J, Sheng Y. Numerical simulation of concrete pumping process and investigation of wear mechanism of the piping wall. Tribology International, 46: 137-144 (2012)

[24] Uzi A, Ben Ami Y, Levy A. Erosion prediction of industrial conveying pipelines. Powder Technology, 309: 49-60 (2017) 
[25] Powell M S, Weerasekara N S, Cole S, LaRoche R D, Favier J. DEM modelling of liner evolution and its influence on grinding rate in ball mills. Minerals Engineering, 24: 341-351 (2011)

[26] Varga M, Goniva C, Adam K, Badisch E. Combined experimental and numerical approach for wear prediction in feed pipes. Tribology International, 65: 200-206 (2013)

[27] Jafari A, Saljooghi Nezhad V. Employing DEM to study the impact of different parameters on the screening efficiency and mesh wear. Powder Technology, 297: 126-143 (2016)

[28] Chu K W, Kuang S B, Yu A B, Vince A, Barnett G D, Barnett P J. Prediction of wear and its effect on the multiphase flow and separation performance of dense medium cyclone. Minerals Engineering, 56: 91-101 (2014)

[29] Forsström D, Jonsén P. Calibration and validation of a large scale abrasive wear model by coupling DEM-FEM: Local failure prediction from abrasive wear of tipper bodies during unloading of granular material. Engineering Failure Analysis, 66: 274-283 (2016)

[30] Brilliantov N V, Spahn F, Hertzsch J-M, Pöschel T. Model for collisions in granular gases. Physical Review E, 53: 5382-5392 (1996)

[31] Hertzsch J-M, Spahn F, Brilliantov N V. On Low-Velocity Collisions of Viscoelastic Particles. J. Phys. II France, 5: 1725-1738 (1995)

[32] Gear C W. Numerical initial value problems in ordinary differential equations Englewood Cliffs, New Jersey: Prentice Hall, 1971.

[33] Christoph Kloss, Christoph Goniva, Alice Hager, Stefan Amberger, Stefan Pirker. Models, algorithms and validation for open source DEM and CFD-DEM. Progress in Computational Fluid Dynamics An International Journal, 12(2/3): 140-152 (2012)

[34] Ayachit U. "The ParaView Guide: A Parallel Visualization Application,” ed: Kitware, ISBN 978-1930934306, 2015.

[35] Burstein G T, Sasaki K. Effect of impact angle on the slurry erosion-corrosion of 304L stainless steel. Wear, 240: 80-94 (2000)

[36] Sheldon G L, Kanhere A. An investigation of impingement erosion using single particles. Wear, 21: 195-209 (1972)

[37] Chandel S, Singh S N, Seshadri V. Experimental Study of Erosion Wear in a Centrifugal Slurry Pump Using Coriolis Wear Test Rig. Particulate Science and Technology, 30: 179-195 (2012)

[38] Gok M S, Gencel O, Koc V, Kuchuk Y, Cay V V. Effect of abrasive particle sizes on abrasive wear of ceramic coatings sprayed by plasma process. Powder Metallurgy and Metal Ceramics, 50: 322-330 (2011)

[39] Trevisiol C, Jourani A, Bouvier S. Effect of martensite volume fraction and abrasive particles size on friction and wear behaviour of a low alloy steel. Tribology International, 113: 411-425 (2017)

[40] Ojala N, Valtonen K, Kivikytö-Reponen P, Vuorinen P, Siitonen P, Kuokkala V T. Effect of test parameters on large particle high speed slurry erosion testing. Tribology-Materials, Surfaces \& Interfaces, 8: 98-104 (2014)

[41] Clark H M, Hartwich R B. A re-examination of the 'particle size effect' in slurry erosion. Wear, 248: 147-161 (2001)

[42] Gandhi B K, Borse S V. Effects of particle-size and size distribution on estimating erosion wear of cast iron in sandwater slurries. Indian Journal of Engineering \& Materials Sciences, 9: 480-486 (2002)

[43] Pagalthivarthi K V, Furlan J M, Visintainer R J. Effect of Particle Size Distribution on Erosion Wear in Centrifugal Pump Casings. V01CT20A005 (2013)

[44] Sevim I, Eryurek B. Effect of abrasive particle size on wear resistance in non-heat-treated steels. Koveve Materialy, 43: 158-168 (2005)

[45] Patil M S, Deore E R, Ramchandra, Jahagirdar S, Patil S V. Study of the Parameters Affecting Erosion Wear of Ductile Material in Solid-Liquid Mixture. Proceedings of the World Congress on Engineering: (2011)

[46] Wiederhorn S M, Hockey B J. Effect of material parameters on the erosion resistance of brittle materials. Journal of Materials Science, 18: 766-780 (1983)

[47] Aquaro D, Fontani E. Erosion of Ductile and Brittle Materials. Meccanica, 36: 651-661 (2001)

[48] Antypov D, Elliott J A. On an analytical solution for the damped Hertzian spring. EPL (Europhysics Letters), 94: 50004 (2011)

[49] Lyczkowski R W, Bouillard J X. State-of-the-art review of erosion modeling in fluid/solids systems. Progress in Energy and Combustion Science, 28: 543-602 (2002)

[50] Azimian M, Bart H-J. CFD simulation and experimental analysis of erosion in a slurry tank test rig. EPJ Web of Conferences, 45: 01009 (2013)

[51] Okonkwo P, Mohamed A M A, Ahmed E. Influence of particle velocities and impact angles on the erosion mechanisms of AISI 1018 steel. Advanced Materials Letters, 6: 653-659 (2015)

[52] Stevenson A N J, Hutchings I M. Scaling laws for particle velocity in the gas-blast erosion test. Wear, 181-183: 56-62 (1995)

[53] Lindsley B A, Marder A R. The effect of velocity on the solid particle erosion rate of alloys. Wear, 225-229: 510-516 (1999)

[54] Rodríguez E, Flores M, Pérez A, Mercado-Solis R D, González R, Rodríguez J, Valtierra S. Erosive wear by silica sand on AISI H13 and 4140 steels. Wear, 267: 2109-2115 (2009) 


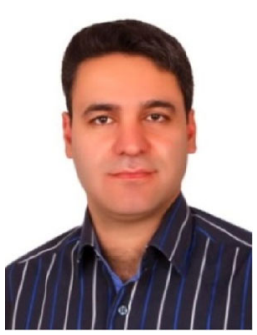

Akbar JAFARI. He received his $\mathrm{Ph} . \mathrm{D}$ degree in mechanical engineering from the University of Tehran in 2011. He started his academic career at Shahid Bahonar University

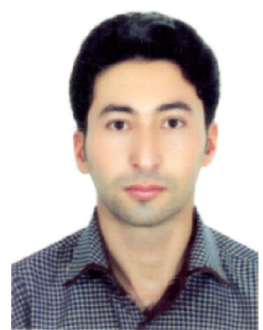

Reza ABBASI HATTANI. He received his bachelor degree in mechanical engineering in 2013 from Shahid Bahonar University of Kerman, Kerman, Iran. After then, of Kerman in 2007, then joined Sirjan University of Technology in 2011 and his current position is associate professor. His research area covers finite element method, discrete element method, wear and non-classical elasticity.

he obtained his master degree in mechanical engineering in 2016 from Sirjan University of Technology, Sirjan, Iran. His research interests include experimental wear analysis and discrete element method. 